\title{
Title
}

\section{Sub-tomogram averaging in RELION}

\author{
Authors \\ Tanmay A.M. Bharat* \& Sjors H.W. Scheres*
}

\section{Affiliations}

Structural Studies Division, MRC Laboratory of Molecular Biology, Cambridge CB2 0QH, United Kingdom

*Correspondence to: tbharat@mrc-lmb.cam.ac.uk or scheres@mrc-lmb.cam.ac.uk 


\begin{abstract}
Electron cryo-tomography (cryo-ET) and sub-tomogram averaging allow structure determination of macromolecules in situ, and are gaining in popularity for initial model generation for singleparticle analysis. We describe herein, a protocol for sub-tomogram averaging from cryo-ET data using the RELION software. We describe how to calculate newly developed three-dimensional models for the contrast transfer function and the missing wedge of each sub-tomogram, and how to use these models for regularized-likelihood refinement. This approach has been implemented in the existing workflow for single-particle analysis, so that users may conveniently tap into existing capabilities of the RELION software. As example applications, we present analyses of purified hepatitis B capsid particles and $S$. cerevisiae $80 \mathrm{~S}$ ribosomes. In both cases, we show that following initial classification, sub-tomogram averaging in RELION allows de novo generation of initial models, and provides high-resolution maps where secondary structure elements are resolved.
\end{abstract}




\section{Introduction}

Imaging macromolecular complexes that are frozen in a thin layer of vitreous ice using an electron microscope (cryo-EM) is rapidly gaining in popularity. A single cryo-EM image may contain 2D projections of many copies of the same complex in different orientations, and these $2 \mathrm{D}$ projections may be combined in a $3 \mathrm{D}$ reconstruction of its scattering potential. This technique, which is known as single-particle analysis, has recently undergone significant progress with the development of highly efficient direct-electron detectors and improved image processing software. Notably, this technique now allows near-atomic resolution structure to be calculated without the need for crystallisation and from as little as 10-100 $\mu \mathrm{g}$ of purified material (Bai et al., 2015; Cheng, 2015).

Alternatively, in electron tomography (ET) multiple images are taken of the same sample region at different tilt angles in the microscope. From such a series of tilted images, a 3D reconstruction, or tomogram, may be obtained of a unique $3 \mathrm{D}$ object such as an entire cell (Baumeister, 2002). Thereby, this technique provides the unique possibility to image macromolecular complexes in their native environment. Moreover, if many copies of a complex of interest are present in tomograms, then the reconstructed 3D density corresponding to each complex may be computationally extracted, and the resulting 3D 'sub-tomograms' may be averaged together to increase the signal-to-noise ratio and thereby produce a higher resolution 3D structure (Briggs, 2013). This technique is called sub-tomogram averaging, and it has been successfully applied in numerous cases to reveal biological structures in situ or in environments that are otherwise not amenable to single-particle analysis (Beck et al., 2007; Briggs et al., 2009; Förster et al., 2005; Grünewald et al., 2003; Lin et al., 2014; Nicastro et al., 2006).

To date, the use of sub-tomogram averaging is not as widespread as that of single-particle analysis. An important limitation of sub-tomogram averaging is that the best resolved structures by this technique are markedly lower in resolution than those from single-particle analysis (Briggs, 2013). Another reason may be that tomographic data collection is slower, and subtomogram averaging requires more complicated image processing, since tomographic reconstruction needs to be followed by alignment and classification of the sub-tomograms. Still, the advantage of being able to study macromolecules in situ remains extremely attractive. This is powerfully illustrated by the recent application of sub-nanometer resolution cryo-ET subtomogram averaging to the HIV-1 capsid (Schur et al., 2015) and to membrane-bound ribosomes (Pfeffer et al., 2015). Further developments of both experimental data acquisition procedures (Rigort and Plitzko, 2015) and image processing algorithms (Hrabe et al., 2012) will continue to drive this technique towards higher resolutions and wider applicability.

Recently, we introduced a new image processing approach to sub-tomogram averaging (Bharat et al., 2015) that is based on regularized likelihood optimization in the RELION program 
(Scheres, 2012a, b). This program was originally designed for single-particle analysis and has been used to calculate numerous near-atomic resolution structures (Bai et al., 2015). Because the sub-tomogram averaging approach in RELION was modelled on the single-particle workflow, existing RELION users will find many similarities (Figure 1). The main deviation from the single-particle workflow lies in the generation of a 3D model for the information transfer in each sub-tomogram, which is used to compensate for both the missing wedge as well as the effects of the contrast transfer function (CTF) in the tilt series images (Bharat et al., 2015). A significant effort was made to build on existing tools inside RELION, rather than writing new tools specifically for sub-tomogram averaging. This facilitates transitioning between sub-tomogram averaging and single-particle analysis, and thus naturally supports a hybrid approach (Bartesaghi et al., 2012; Bharat et al., 2014; Bharat et al., 2012).

In this protocol we describe the practical use of RELION for sub-tomogram averaging. Our approach complements various single-particle analysis software packages that also offer functionalities for sub-tomogram averaging, e.g. (Heymann and Belnap, 2007; Tang et al., 2007), as well as multiple specialized packages for sub-tomogram averaging, e.g. (Castano-Diez et al., 2012; Förster et al., 2005; Hrabe et al., 2012; Huiskonen et al., 2010; Kremer et al., 1996; Nicastro et al., 2006). As many structure determination projects in practice resort to a combination of different software packages, we will explicitly indicate those points in the workflow that are likely points of conversion between alternative approaches. Recommended procedures for single-particle analysis in RELION are described in detail in the online documentation (the RELION wiki: http:/www2.mrc-lmb.cam.ac.uk/relion). Using the protocol described here together with the online documentation, novice users should be able to conduct sub-tomogram averaging for their own project using RELION. We assume basic familiarity with Unix/Linux based systems, and the ability to run provided scripts from the command-line. 


\section{Materials}

\section{Equipment setup}

- A computer or a computing cluster with at least $64-128$ processors, $120 \mathrm{~Gb}$ of shared memory, and $1 \mathrm{~Tb}$ of disk space.

- A RELION installation (version 1.4 or newer; available for free from http://www2.mrclmb.cam.ac.uk/relion).

- A Python installation (version 2.6 or newer; available from https://www.python.org)

- An IMOD installation (version 4.7 or newer; available for free from http://bio3d.colorado.edu/imod/) 


\section{Procedure}

Steps 1-6 explain how input files and directories should be arranged for RELION sub-tomogram averaging.

1| The directory from which all RELION commands and the graphical-user-interface (GUI) is launched will be known as the project directory ( ./ ). We recommend making a folder inside the project directory that is called ./Tomograms / where all the input data is stored.

2| To enter the RELION workflow, we assume that tomograms have been generated in MRC format (Crowther et al., 1996). Tomogram calculation is not done inside RELION, but relies on software packages like IMOD (Kremer et al., 1996), Tomo3D (Agulleiro and Fernandez, 2011), pyTOM (Hrabe et al., 2012) or Bsoft (Heymann and Belnap, 2007). In the examples below, we used the IMOD package for tilt series alignment, and we used Tomo3D for tomographic reconstruction. Generate a sub-directory in the ./Tomograms/ directory with the name of each tomogram in the data set, and copy all tomograms to their respective sub-directories. For example, if there are two tomograms in the data set, then the locations of those tomograms should be as follows -

./Tomograms/tomogram1/tomogram1.mrc

./Tomograms/tomogram2/tomogram2.mrc

$3 \mid$ The coordinates of the centres of all macromolecular complexes, or particles, in a tomogram should be saved in the same sub-directories as the corresponding . mrc file. The coordinate files should have the suffix . coords and the prefix should be the same as the prefix of the tomogram name. In the results presented here we either used the MolMatch software for template matching (Förster et al., 2005) or manually picked particles from IMOD (Kremer et al., 1996). In our twotomogram example the name and location of the coordinate files should be -

. / Tomograms/tomogram1/tomogram1. coords

. / Tomograms/tomogram2/tomogram2. coords

The coordinates within each file should be written out in a three-column ASCII format, corresponding to the $\mathrm{X}, \mathrm{Y}, \mathrm{Z}$ position of the centre of each sub-tomogram in pixels. Each coordinate file should contain as many lines as there are particles in the tomogram. For example

$\begin{array}{lll}100.0 & 355.0 & 200.0 \\ 2034.0 & 1100.0 & 561.0\end{array}$


$3011.0 \quad 2539.0 \quad 321.0$

4| For CTF estimation, the aligned tilt series should also be placed in the same sub directories. For each tomogram, this should be a single MRC stack, and the suffix of the files should be .mrcs. It is important that this is the exact same aligned tilt series as the one used for tomographic reconstruction. Again, in our example these should be called -

- /Tomograms/tomogram1/tomogram1.mrcs

. / Tomograms/tomogram2/tomogram2.mrcs

5| Along with the aligned stack, also provide the final tilt angles from IMOD in a separate text file. Each line in these text files should contain one number corresponding to the final tilt angle assigned to that image during alignment. The order of the lines should correspond to the order of the images in the aligned stack.

59.44

56.44

53.43

Copy these angles files into the same directory.

. / Tomograms/tomogram1/tomogram1.tlt

. /Tomograms/tomogram2/tomogram2.tlt

If .tlt files with angle values are not provided, then the tilt angles will be read from the extended header of the .mrcs file, if this exists, in step 9 (see below).

6| For each aligned tilt series, a text file should be created that lists the tilt angles and the accumulated radiation for each image in the tilt series. This information will be used to calculate a dose-dependent 3D CTF model (Figure 2A-B) that also accounts for radiation-induced damage of the specimen. This text file should have as many lines as there are images in the tilt series. The text file should have two columns: the first one for the refined tilt angle (after tilt-series alignment), and the second one for the total accumulated dose in $\mathrm{e}^{-} / \AA^{2}$ prior to collecting that image. If you provide the nominal tilt angles rather than the refined tilt angles, the python setup script in step 9 will assign accumulated dose values to the closest refined tilt angle from the . $t$ t file. For example -

$\begin{array}{ll}61.1 & 0.0 \\ 57.3 & 2.0 \\ 54.2 & 4.0\end{array}$

Save these files in the same sub-directories with the suffix . order - 
./Tomograms/tomogram1/tomogram1.order

./Tomograms/tomogram2/tomogram2.order

7| Generate a RELION-type metadata file in the STAR format (Hall, 1991; Scheres, 2012b), called all_tomograms.star, using the command line -

relion_star_loopheader rlnMicrographName > all_tomograms.star

ls./Tomograms/*/*.mrc $>$ all_tomograms.star

The output file lists all the tomograms in the data set, and should have the format -

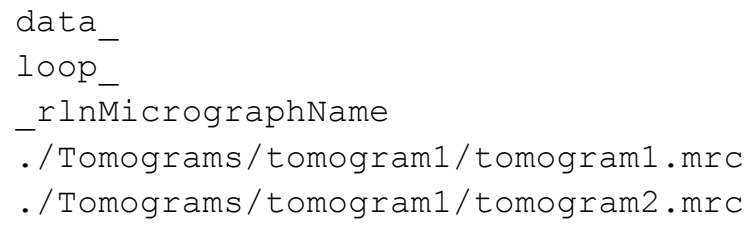

Steps 8-14 illustrate how to calculate the 3D CTF models for each sub-tomogram. To facilitate routine application of these steps, we provide a single python script called relion_prepare_subtomograms.py on the RELION wiki. This script takes the all_tomograms.star file as input, and carries out steps 8-14, depending on the options that the user specifies in its header. For specialized applications or in case of troubleshooting, any of the steps 8-14 may be carried out independently.

8| CTF correction depends on the estimation of a defocus value for every image of the tilt series (i.e. for all the individual images in the .mrcs stacks mentioned above). We do this by calling CTFFIND (Mindell and Grigorieff) through the wrapper provided in RELION: relion_run_ctffind. Executing this program will be done automatically by the relion_prepare_subtomograms.py script. Input parameters for CTFFIND should be changed in the header of the python script. Specify inputs like voltage, spherical aberration and detector pixel size based on your data collection experiment.

\section{? TROUBLESHOOTING}

9| Next, run the provided python script from the RELION project directory -

python relion_prepare_subtomograms.py 
As in single-particle analysis, this script will create a ./Particles/Tomograms/ directory with one sub-directory per tomogram. Following CTF estimation using CTFFIND, in each tomogram sub-directory . star files corresponding to the CTF model for each sub-tomogram will be written out. A particles_subtomo.star file that lists all the sub-tomograms as well as their corresponding 3D CTF models will also be written to the project directory. This file will be the input for 3D classification and 3D auto-refinement in RELION.

\section{? TROUBLESHOOTING}

10| It is important to inspect the diagnostic output of CTFFIND for every image of every tilt series in the data set (Figure 2C-E). This may be conveniently done using the 'Display' button in the RELION GUI (Figure 3), and selecting the output_ctffind.star file for each tomogram, e.g. . /Tomograms/tomogram1/ctffind/tomogram1_ctffind.star. If CTF estimation failed for some images (see Figure 2E), then relion_run_ctffind should be executed again for that image with different parameters. We recommend that either the entire python script should be run again (step 9), or users can look in the relion_subtomo_commands.txt file for the relevant commands for individual tilt series and run them again. Alternatively, one could use a CTF estimation program from an alternative program, possibly one that allows manual steering like EMAN2's e2ctf.py (Tang et al., 2007), to find a defocus that fits the observed power spectrum of the image. In that case, the resulting defocus value should be inserted manually into the output _ctffind.star file by changing the corresponding values using a text file editor.

\section{? TROUBLESHOOTING}

11| (Steps 11-13 relate to extra options provided to improve CTF estimation accuracy) For flat samples, the effective thickness of the ice that the electron beam passes through increases with increasing tilt angle. This results in lower signal-to-noise ratios for the higher tilt images, which may preclude reliable CTF estimation. If this is the case, the average defocus measured for the lower tilt images may be applied to the higher tilt images, especially if the applied defocus is stable throughout the tilt series (Schur et al., 2015). This may be done conveniently by setting the UseonlyLowertiltDefoci variable to True in the header of the relion_prepare_subtomograms.py script and providing a threshold value for the lower tilt.

12| Another way to estimate the CTF parameters of higher tilt images more accurately is to acquire two extra images for each setting of the stage in the tilt series. We collected these images along the direction of the tilt axis, and spaced equally on either side (Bharat et al.; Eibauer et al., 
2012) of the region of interest. If such images were collected, then they should be saved in MRC format with a . trial suffix in the relevant tomogram sub-directories -

- /Tomograms/tomogram1/tomogram1.trial

. / Tomograms/tomogram2/tomogram2.trial

Note that the number of images in the trial stack should be exactly double the number of images in the original tilt-series stack and the order of the images should be the same as the aligned tilt series $\left(60^{\circ}, 60^{\circ}, 57^{\circ}, 57^{\circ}\right.$ and so on). We do not recommend collecting a single extra image one side of the region of interest because there could be a systematic focus offset between the extra image and the tilt series image. In order to run CTFFIND on these extra images, one should set the UseTrialsForCtffind variable in the relion_prepare_subtomogram.py script to True.

13| The last method to improve the 3D CTF model is to apply a linear, dose-dependent B-factor to the data (also see (Bharat et al., 2015)). Based on observations made for single-particle data sets (Scheres, 2014), we increased the B-factor by $4 \AA^{2}$ for each $1 \mathrm{e}^{-} / \AA^{2}$ accumulated dose for both examples described in this paper. Users may want to select a different value depending on the radiation sensitivity of their specimen. To set this parameter, change the Bfactor variable in the header of the python script. Note that the tilt-dependent scale factor and the positiondependent defocus of each particle will be calculated automatically for every image in the tilt series.

14| The command to reconstruct each 3D CTF model is written out in a run script called do_all_reconstruct_ctfs.sh, which is automatically generated in step 9. This script should be run from the RELION project directory -

. /do_all_reconstruct_ctfs.sh 200

In the above command, the parameter ' 200 ' determines the size of the generated CTF volumes in pixels. This should be set to the same values as the "Particle box size" in step 15, i.e. the size of the extracted sub-tomograms. The above script contains multiple commands, and may thus be split into shorter text files for convenient parallelization.

15| Next, one extracts every particle into a sub-tomogram by performing a 3D window operation on the corresponding tomogram. On the "General" job-type of the RELION GUI (Figure 3), provide the tomogram pixel size in $\AA$, and the diameter of a spherical mask (in $\AA$ ) that will be used for calculating the average and standard deviation of the background pixel values. Make 
sure this mask is slightly larger than the longest diameter of the particle. Then, on the "Particle extraction" job-type provide the all_tomograms.star file (from step 7) as "micrograph STAR file"; set the "Coordinate-file suffix" to .coords; and "rootname" should be set to the same as the RootName variable in the relion_prepare_subtomograms.py script. The "Particle box size" (which is given in pixels) should be set to reflect $150-200 \%$ of the particle's longest dimension. It is recommended to invert the contrast of the sub-tomograms if the particles in the tomogram are black. The re-scaling option may be used to downscale the extracted sub-tomograms, which will save computational resources if the tomograms were taken with a smaller pixel size than necessary for the target resolution. Running this job (by pressing the "Run!" button on the GUI) will write out the sub-tomograms as individual .mrc files in the ./Particles/Tomograms/tomogram?/ directories that were created by the python setup script in step 9.

16| Along with ordinary sub-tomograms (which are 3D volumes), 2D projections of all subtomograms (along the Z-axis) may also be calculated by again using the 'Particle extraction' job-type. This is done by running the same job as in step 15, but with providing the extra option --project3d on the 'Additional arguments' line of the 'Running' tab. By setting the 'Extract rootname' on the 'I/O' tab to subtomo_proj2d, an additional .star file called ./subtomo_proj2d.star will be generated, which lists the $2 \mathrm{D}$ projections of all subtomograms.

17| The 2D projections may be used in reference-free 2D classification of the data, much like one would use in single-particle analysis, which is a computationally cheaper alternative to classification of the sub-tomograms (Bharat et al., 2015; Yu et al., 2013). On the 'I/O' tab of the '2D classification' job-type, provide the ./subtomo_proj2d.star as the input .star file, and set the 'Output rootname' for example to Class2D/run1. The number of classes will depend on the number of sub-tomograms and the expected heterogeneity in the data. As a rule of thumb, we typically use at least on average 30 sub-tomograms per class, and we hardly ever use more than 50-100 classes. Because the 2D projections do not have a CTF model, switch CTF-correction off on the 'CTF' tab. On the 'Optimisation' tab, we typically perform 25 iterations, and use a regularisation parameter of 2 for 2D classification (Scheres, 2012a). Typically, we mask particles with zeros, and provide a limit on the resolution of around 10-15 $\AA$ to include in the expectation (E-) step of the algorithm. On the 'Sampling' tab, the default parameters are suitable for most cases. Executing this job through the 'Run!' button will result in multiple output files for each iteration in the $/ \mathrm{Class} 2 \mathrm{D}$ directory. The final class averages are stored in a mrcs stack file called./Class2D/run1_it025_classes.mrcs. The ./Class2D/run1_it025_model.star file contains information about the final classes, such as their relative size and the estimated resolution of each class average. 
18| The resulting 2D class averages from step 17 may be visualized using the 'Display' button on the GUI and selecting the Class2D/run1_it025_model.star file. In the subsequent pop-up window, it is useful to reverse-sort the classes on rlnclassDistribution, which will place the largest class averages on the top of the display window. At this point, the user needs to select good-looking class averages (which reveal recognizable protein features, e.g. see Figure 4A-B) by double-clicking the class averages, which will put a red border around the image. A new . star file with only the particles that correspond to the good classes may then be saved by rightclicking the display window, and selecting the option 'Save STAR with particles from the selected classes' option from the pop-up menu as subtomo_proj2d_sel.star .

19| If desired, users may also use the selected 2D classes to write a . star file containing only the corresponding sub-tomograms. We again provide a python script on the RELION wiki to facilitate this. Run this script from the project directory -

python relion_2Dto3D_star.py subtomo_proj2d_sel.star particles_subtomo.star

This script will take two input .star files; the first is the subtomo_proj2d_sel.star file containing selected particles from step 18 and the second is the particles_subtomo.star file containing all sub-tomograms from step 9. This script will write out the subset of the subtomograms selected in step 18 into output_2Dto3D.star.

20| Once a subset of particles has been identified using steps $18-19$, this subset may be used to get a sub-tomogram averaging structure using the '3D auto-refine' job-type. The output rootname on the 'I/O' tab could for example be ./Refine3D/run1. Whereas single-particle analysis requires an input 3D reference map, sub-tomogram averaging in either the $3 \mathrm{D}$ autorefine or 3D-classification (see next step) job-types may be performed without an initial reference by stating None at the 'Reference map' entry on the 'Reference' tab. In the case an external 3D reference is provided, one also needs to indicate whether it is on the correct absolute greyscale. In general, maps created by RELION from the same data will be on the correct greyscale, whereas maps coming from elsewhere may not be. On the same tab, one also needs to provide the resolution of an initial low-pass filter that will be applied to the input 3D map. To prevent model bias, we typically use relatively harsh filters, e.g. in the range of 40-100 A. On the 'CTF' tab, one can turn on CTF correction (provided steps 8-14 were performed). If CTFcorrection is performed and a $3 \mathrm{D}$ map is provided as initial reference, then one also needs to indicate whether the input map has been CTF-corrected. Maps from RELION may be CTFcorrected (depending on whether CTF correction was performed in previous runs), and also for maps that were generated from an atomic model one needs to indicate they have been CTF- 
corrected (i.e. to reflect that they don't suffer from CTF artefacts). On the 'Optimisation' tab, we typically use similar options as for 2D classification runs, with the exception of the regularisation parameter, which is typically set to 4 for 3D auto-refine and classification (see next step). For the options in the 'Auto-sampling' tab, the angular and offset sampling rates and ranges will only be used in the first several iterations. After that the auto-sampling algorithm will automatically use finer samplings and smaller ranges until the refinement converges (Scheres, 2012b). The default parameters will be suitable for most projects, perhaps with the exception of particles with icosahedral symmetry, for which initial angular sampling rates of 3.7 degrees, and local angular searches from 0.9 degrees may yield better results. Note that the auto-refinement will divide the data into two random half-sets, each of which will be refined independently, so that in the next step "gold-standard" resolution estimates may be calculated (Henderson et al., 2012; Scheres and Chen, 2012). Executing this job will output multiple files for each iteration in the ./Refine3D directory. The final ./Refine3D/run1_half[1,2]_class001_unfil.mrc files will be used in the post-processing as described in step 24 .

21| Once a 3D reconstruction has been obtained using sub-tomogram averaging, the subtomograms may be classified using the '3D classification' job-type on the GUI to detect different conformational states of the specimen. On the 'I/O' tab select the original particles_subtomo.star file, or the output_2Dto3D.star from the 2D classification described in step 19. The output rootname could for example be Class3D/run1. Because of computational costs, we often use fewer classes for 3D classification than for 2D classification, with typical values in the range of 3-10. On the 'Reference' tab, use the final reconstruction from step 20 (./Refine3D/run1_class001.mrc) as the reference map. This map is now on the correct absolute greyscale, and a similar initial low-pass filter as in step 20 may be applied. On the 'CTF' tab indicate that the reference has been CTF-corrected (if this was indeed done in step 20). On the 'Sampling' tab, the default parameters are again suitable for most projects. Executing this job will result in multiple output files for each iteration in the ./Class3D directory. The final reconstructions for each class are stored in .mrc files called ./Class3D/run1_it025_class0??.mrc . The ./Class3D/run1_it025_model.star file contains information about the final classes, such as their relative size and the estimated resolution of each reconstruction.

22| The user again needs to decide which of the classes look good. The 'Display' button on the GUI may be used to show 2D slices through each of the 3D maps (e.g. select /Class3D/run1_it025_class001.mrc to visualize the first class). Visualization of all classes together in UCSF Chimera (Pettersen et al., 2004) is also useful. Once good classes have been identified, one should select the ./Class3D/run1_it025 model.star file from the 'Display' 
button on the GUI to display (central slices through) the maps of all classes. A . star file with the particles belonging to the good classes can then be saved in the same way as in step 18 .

23| Sometimes, performing steps 20-22 multiple times, where selected classes from one run are re-classified in a next run, helps to obtain more homogeneous classes. Thus, each class as identified in steps 21-22 should again be refined to high-resolution using the $3 \mathrm{D}$ auto-refinement as described in step 20.

24| After completion of the final 3D auto-refinement for each class, the 'Post-processing' jobtype is used to obtain resolution estimates that have been corrected for the influence of a solvent mask (Chen et al., 2013), to calculate for the modulation transfer function (MTF) of the detector, and to sharpen the final map. On the 'I/O' tab, one of the two unfiltered half-maps should be provided as./Refine3D/run1_half1_class001_unfil.mrc; the output rootname could be set to run1_post. On the 'Mask' tab, the user may provide parameters for the calculation of an automated solvent mask (automask). The average of the two half-maps will be binarized at the specified initial threshold; extended by the provided number of pixels; and finally a cosineshaped soft edge with the specified width will be added to the mask. The initial threshold value should be chosen such that the automask does not contain isolated white regions in the solvent area. Often a good estimate for the initial threshold value is the threshold at which a display of the ./Refine3D/run1_class001.mrc map in UCSF Chimera is noise-free in the region around the particle. Once a suitable automask has been created, it can also be provided as input on the 'Mask' tab instead of calculating a new automask in subsequent Post-processing runs. On the 'Sharpen' tab, a MTF curve for the detector may be provided. Curves for some detectors may be downloaded from the RELION wiki. For other detectors, the manufacturer may provide MTF curves. If no curve is available, this entry may also be left empty, in which case MTF-correction will be emulated by additional B-factor sharpening. For maps with resolutions beyond 9-10 A, automated B-factor sharpening (Rosenthal and Henderson, 2003) may be performed. Alternatively, a user-defined value may be provided. Typically, the option to skip FSCweighting on the 'Filter' tab is not used. Execution of this step will generate the final map (./Refine3D/run1_post.mrc), the automask (./Refine3D/run1_post_automask.mrc) and a .star file with the applied B-factor, the resolution estimate and the corrected FSC curve (./Refine3D/run1_post.star). In addition, the corrected FSC curve will be written out as a file called.Refine3D/run1_post_fsc.xml, which can be directly uploaded to the EMDB.

\section{? TROUBLESHOOTING}


25| Because many macromolecular complexes are inherently flexible, even after classification data sets will often still contain some extent of structural heterogeneity. This will lead to local variations in resolution in the refined map. To estimate local resolution variations, the 'Localresolution' job-type implements a wrapper to the ResMap program (Kucukelbir et al., 2014). On the 'I/O' tab, again provide one of the two unfiltered half-maps, and provide the range and step size of the resolutions to be tested by ResMap. One typically does not change the default Pvalue. ResMap will provide much more reliable resolution estimates if one provides a suitable mask. The automask calculated in the previous step typically performs well. The 'Run!' button will launch the GUI from the ResMap programs. In most cases one can just hit 'Continue' inside ResMap, i.e. without adjusting any of its parameters. The result is a file called ./Refine3D/run1_half1_class001_unfil_resmap.mrc, which can be used inside UCSF Chimera to colour the ./Refine3D/run1_post.mrc map, using menu options Tools -> Surface Color -> by volume data value.

26| When a refined map still shows signs of large amounts of structural heterogeneity, i.e. it contains regions of relatively low local resolution, it may be useful to perform a focused classification on that specific region. In that case, one inputs the ./Refine3D/run1_data.star file into a new 3D classification run as explained under step 21. In this run, one could use a solvent mask that is only white in the disordered region. Also, one could either skip orientational searches (through the corresponding option on the 'Sampling' tab), or one could perform relatively fine, but local angular searches around the input orientations. Thereby, one may iterate two or more times through steps 20-25. 


\section{Timing}

The time taken for the procedure depends approximately linearly on the number of tomograms, the number of sub-tomograms and (inversely so) on the number of processors used on the cluster. Here, for the HBV capsids, we analyzed a dataset containing 15 tomograms (each occupying $\sim 75 \mathrm{~Gb}$ of hard disk space). 1851 capsid particles were extracted in boxes of $240^{3}$ pixels from these tomograms and data was processed on a computing cluster with 4 hyperthreaded 12-core nodes, each with at least $32 \mathrm{~Gb}$ of RAM. CTF volume reconstruction (distributed over 15 cores, 1 per tomogram) took 2 hours. Sub-tomogram extraction and projection of the extracted sub-tomograms took 30 minutes using a single core. $2 \mathrm{D}$ classification of the data in 20 classes took $\sim 3$ hours on 2 nodes. 3D auto-refinement was concluded in 24 hours, using 4 nodes. Postprocessing was performed on a single core in 10 minutes.

For the $80 \mathrm{~S}$ ribosomes, 7 tomograms were used and 3120 ribosomes were extracted in boxes of $200^{3}$ pixels and projected into $2 \mathrm{D}$ in 20 minutes using a single core. CTF volume reconstruction took 30 minutes on all nodes; 2D classification into 20 classes took 30 minutes on 2 nodes; 3D classification into 3 classes took 10 hours on 4 nodes; and 3D auto-refinement took 50 hours on 4 nodes. 


\section{Troubleshooting}

\begin{tabular}{|c|c|c|c|}
\hline Step & Problem & Possible reason & Solution \\
\hline All & $\begin{array}{l}\text { RELION executables not } \\
\text { found }\end{array}$ & $\begin{array}{l}\text { RELION was not installed } \\
\text { correctly }\end{array}$ & Install RELION 1.4 or newer on your system. \\
\hline All & IMOD executables not found & $\begin{array}{l}\text { IMOD is not installed on the } \\
\text { system. }\end{array}$ & Install IMOD version 4.7 or newer on your system. \\
\hline 8 & CTF correction is not desired. & $\begin{array}{l}\text { Target resolution is lower than } \\
\text { spatial resolution of the first } \\
\text { zero of the CTF, or it is } \\
\text { impossible to estimate and } \\
\text { compensate for the CTF. }\end{array}$ & $\begin{array}{l}\text { Set the variable SkipCTFCorrection in the } \\
\text { relion_prepare_subtomograms.py script to } \\
\text { True. In this case a wedge shaped } 3 \mathrm{D} \text { 'CTF' model } \\
\text { will be created that is weighted appropriately } \\
\text { depending on the tilt angle and the accumulated } \\
\text { radiation. }\end{array}$ \\
\hline 9 & $\begin{array}{l}\text { The python setup script } \\
\text { crashes with the complaint } \\
\text { "File not found". }\end{array}$ & $\begin{array}{l}\text { One or more of the input files } \\
\text { are missing. }\end{array}$ & $\begin{array}{l}\text { Please ensure that all the files needed for steps } 1-7 \\
\text { are present in the correct locations. The .tlt file } \\
\text { may be omitted if the mrcs file has the correct } \\
\text { angles stored in its extended header. }\end{array}$ \\
\hline 9 & $\begin{array}{l}\text { The python script exits with } \\
\text { the warning that the number of } \\
\text { tilt angles and the number of } \\
\text { images is different. }\end{array}$ & $\begin{array}{l}\text { Some images were omitted in } \\
\text { the tomogram reconstruction, } \\
\text { but the tilt angle values were not } \\
\text { updated. }\end{array}$ & $\begin{array}{l}\text { Use the tilt angles for only the images that are } \\
\text { included in the aligned tilt series and thus in } \\
\text { tomogram reconstruction. }\end{array}$ \\
\hline 10 & $\begin{array}{l}\text { CTF cannot be estimated for } \\
\text { some images of the tilt series. }\end{array}$ & $\begin{array}{l}\text { Low signal to noise in the image } \\
\text { leading to poor visibility of } \\
\text { Thon rings. }\end{array}$ & $\begin{array}{l}\text { Either follow one of the strategies in steps } 11-13 \text { for } \\
\text { accurate CTF estimation, or remove the offending } \\
\text { images from the aligned tilt series stack. If the latter } \\
\text { option is followed, the tomogram would have to be } \\
\text { regenerated with that image removed. }\end{array}$ \\
\hline 24 & $\begin{array}{l}\text { The post-processing program } \\
\text { complains that the masked } \\
\text { FSC never drops below } 0.8 \text {. }\end{array}$ & $\begin{array}{l}\text { The automasking procedure } \\
\text { yielded an all-black, or } \\
\text { otherwise unsuitable, mask. }\end{array}$ & $\begin{array}{l}\text { Adjust the parameters of the automasking procedure, } \\
\text { or make a mask outside the post-processing } \\
\text { procedure. For the latter, one could use any program } \\
\text { outside RELION, or the relion_mask_create } \\
\text { program. }\end{array}$ \\
\hline 24 & $\begin{array}{l}\text { Even though estimated } \\
\text { resolution of the reconstruction } \\
\text { is high, the reconstruction } \\
\text { looks lowpass filtered with no } \\
\text { high-resolution features }\end{array}$ & $\begin{array}{l}\text { The tomogram reconstruction } \\
\text { was conducted with a SIRT } \\
\text { algorithm. }\end{array}$ & $\begin{array}{l}\text { Try using weighted back projection for tomogram } \\
\text { generation and re-extract the sub-tomograms, or } \\
\text { apply a negative (ad-hoc) B-factor in the post- } \\
\text { processing (step 24). }\end{array}$ \\
\hline
\end{tabular}




\section{Anticipated results}

We illustrate the results of this protocol for two test data sets. The first set comprised 15 tomograms that were collected on a sample of purified hepatitis B virus (HBV) capsids. For this data set, two extra trial images on either side of the region of interest were used for CTF estimation. After optimizing the input parameters for CTFFIND (defocus search range, resolution search range and box size), CTF estimation from these extra images was found to be adequate even at high tilts (Figure 2D). Thus, the arithmetic mean of the defoci were applied to the tilt series image at every tilt. The coordinate files for the HBV capsids were obtained by automated picking using the template matching routines in the program MolMatch (Förster et al.). In addition to true HBV capsids, this program also picked up $10 \mathrm{~nm}$ gold fiducials and other undesirable features. Using both steps 15 and 16, HBV capsid particles were extracted in $240^{3}$ pixel sub-tomograms, as well as the corresponding 2D projections along the Z-axis. 2D classification, as described in steps 17-19 readily separated HBV capsids from false positives of the automated picking procedure (Figure 4A). 3D auto-refinement (step 20), starting from random orientation assignments for all sub-tomograms, followed by post-processing (step 24), yielded a final map to a resolution of $9.4 \AA$ (Figures $4 \mathrm{C}$ and 5A) from 1851 particles where secondary structure elements like $\alpha$-helices were clearly resolved (Figure $5 \mathrm{C}$ ).

The second test data set of 7 tomograms was collected on a sample of purified $80 \mathrm{~S}$ ribosomes from $S$. cerevisiae. We have deposited this data set together with the results described here at the EMPIAR data base (Patwardhan et al., 2014) under accession number EMPIAR10045. The initial tomograms, the corresponding aligned tilt series, as well as the .tlt, .order, .trial and .coords files as described in steps 1-7 are all stored in the ./Tomograms subdirectory of the EMPIAR entry. The results of all other steps are stored in the ./AnticipatedResults subdirectory of the EMPIAR entry. Thereby, novice users can follow the exact steps described above to replicate, and compare with, the results described here. CTF estimation was conducted using the same procedures as for the HBV capsid data set. However, even after optimizing the inputs of CTFFIND, CTF estimation from high-tilt images was inaccurate in some instances. We believe that this is due to increased specimen thickness due to the deposition of a layer of carbon on the grid during sample preparation. Thus, we used the average of the defoci from the lower tilt images and applied this average to all high tilt images in step 11. Manually picked particles were extracted as sub-tomograms, as well as 2D projections along the Z-axis. 2D classification of the projected sub-tomograms revealed multiple different views of the ribosomes, and some small classes that needed to be discarded (Figure 4B). In this case, 3D auto-refinement of the input 3120 particles, again starting from random orientations, followed by post-processing, led to a $13 \AA$ reconstruction (Figures 4D and 5B). Typical features like grooves of RNA helices were clearly visible in this map (Figure 5D). Using the output of the 3D auto-refinement we also conducted 3D classification (as in step 21) of the entire data set into

3 classes (Figure 5E-G). Although we could not identify different ratcheted states of the ribosomes, we identified a small subset of particles (Class 3, Figure 5G) that gave rise to a poor 
average. Removing these particles from the data set and subsequent 3D auto-refinement resulted in a somewhat cleaner output map, albeit at the same measured resolution. To assess local resolution variations in this map, ResMap analysis was performed as explained in step 25. The resulting map shows somewhat lower resolution in the small subunit than in the large subunit (Figure 5H). 


\section{Acknowledgements}

We thank Xiaochen Bai, Israel Sanchez Fernandez and K. Vinothhumar for help with sample preparation; Jake Grimmett and Toby Darling for assistance with high-performance computing; Shaoxia Chen and Christos Savva for assistance with electron microscopy; and Jan Löwe for helpful discussions. This work was supported by funds from EMBO (ALTF 3-2013 to T.A.M.B.) and the UK Medical Research Council (MC_UP_A025_1013 to S.H.W.S.). 


\section{Figures}

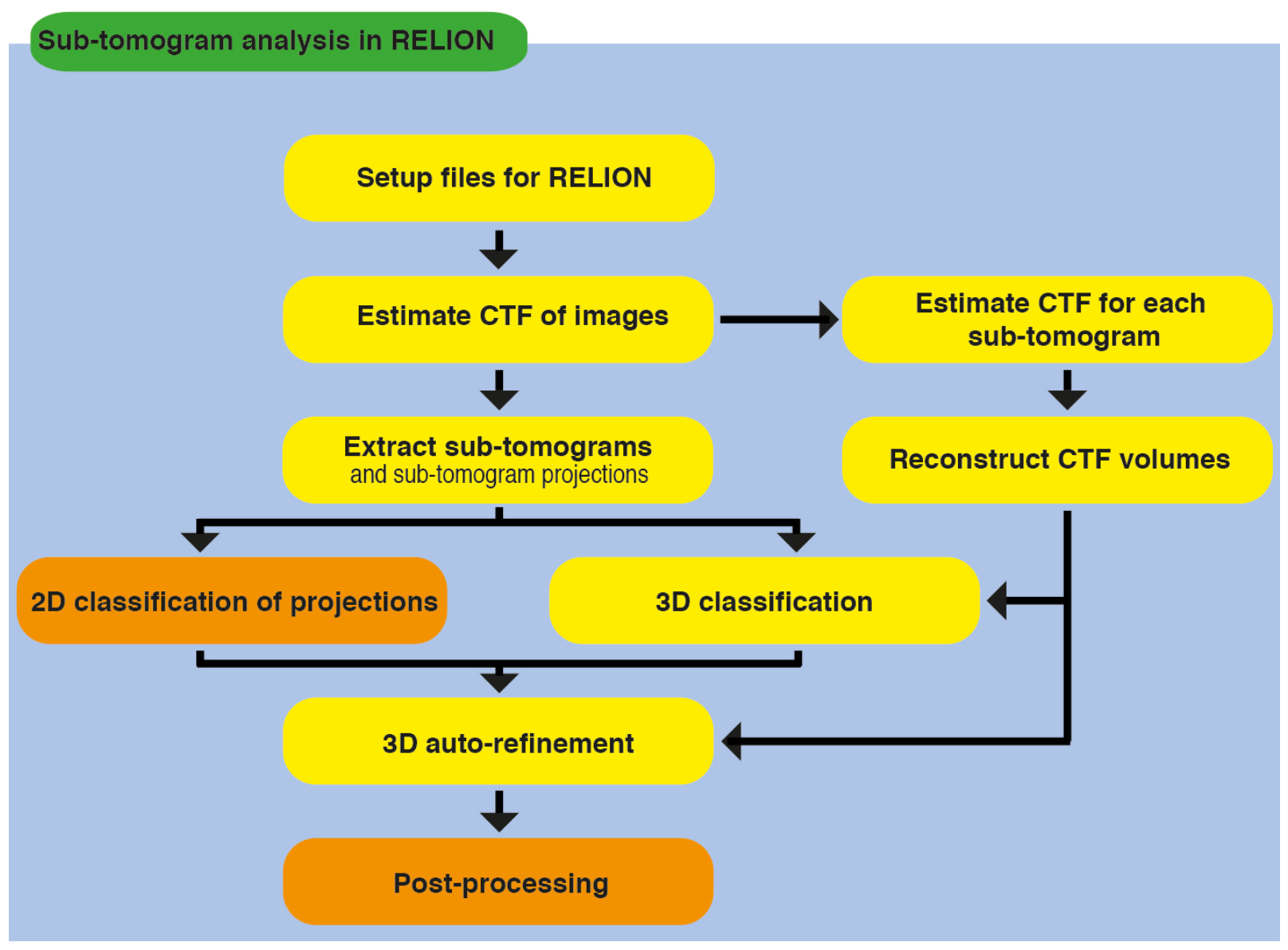

\section{Figure 1. Workflow of the image processing protocol.}

A schematic representation of the recommended workflow for sub-tomogram analysis using RELION presented in this paper. The main difference between single-particle analysis and subtomogram analysis in RELION is related to CTF estimation and the new 3D CTF model. Steps highlighted in orange are unchanged from the single-particle analysis workflow. 


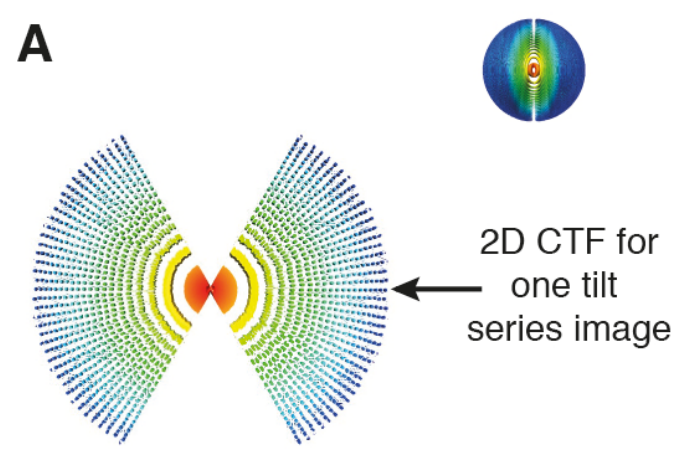

B

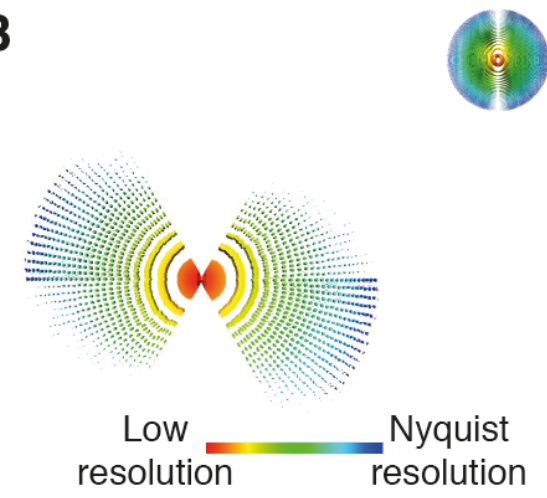

3D CTF Model

Weighted 3D CTF Model

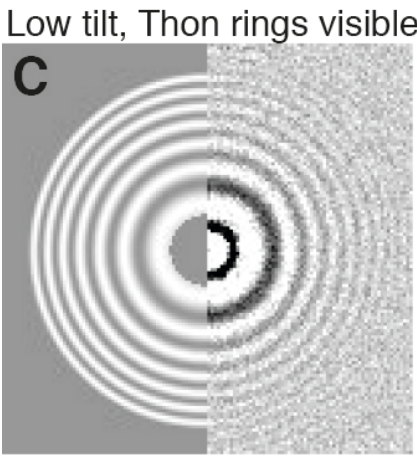

Accurate CTF esimation
High tilt, few Thon rings

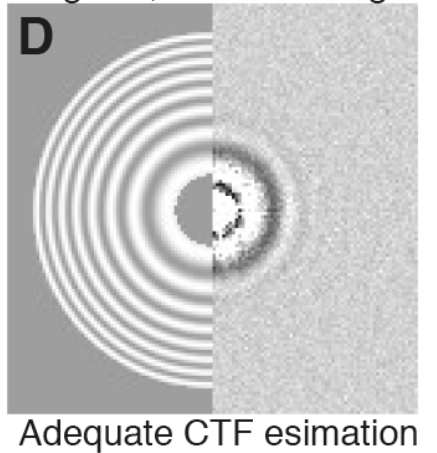

High tilt, no Thon rings

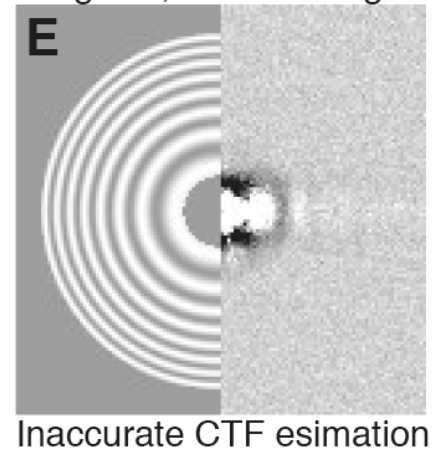

\section{Figure 2. CTF estimation for the 3D CTF model.}

(A) The unweighted 3D CTF model used in RELION. This model also compensates for the missing wedge. (B) The weighted 3D CTF model used in RELION. The weighted model accounts for increase in noise at high tilts, and for radiation-induced damage. (C) A diagnostic output file of CTFFIND3 from a low-tilt tilt series image. There is no visible radiation-induced motion, and many Thon rings are visible making CTF estimation accurate. (D) Corresponding diagnostic file from a high-tilt image. Less Thon rings are visible die to increased specimen thickness. CTF estimation in this case is adequate but not as accurate as C. (E) A diagnostic file from a high-tilt image where no Thon rings are visible. CTF estimation is not possible from this image, and it should either be removed from the tilt series or the data collection strategy should be modified to include the recording of additional images for CTF estimation on either side of the target region (Bharat et al., 2015). 


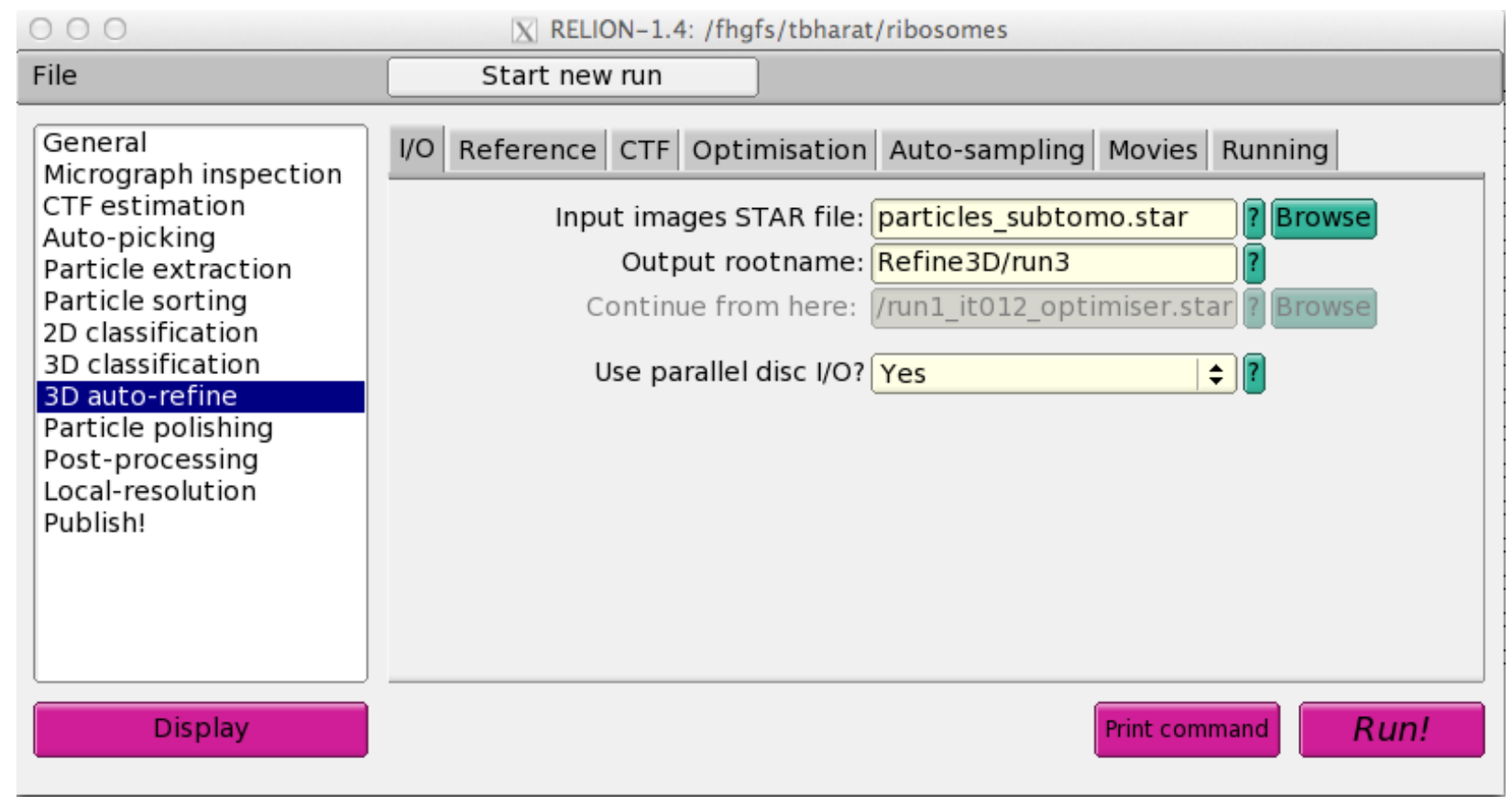

Figure 3. The RELION graphical user interface.

After CTF parameters have been estimated for each particle in each image of the tilt series and 3D CTF models have been reconstructed, the actual tasks of sub-tomogram analysis may all be performed using the RELION graphical user interface. The 3D auto-refine page of this user interface is shown. The white column on the left shows different 'job-types', which are ordered according to the natural workflow from top to bottom. On the main panel, the '3D auto-refine' job-type is shown. This job-type has tabs for "I/O", "Reference", "CTF", "Optimization", "Auto-sampling", "Movies", and "Running" where users should enter the input parameters as described in the main text. The "Display", "Print command" and "Run!" buttons are used to view images, commands and launch jobs, respectively. 

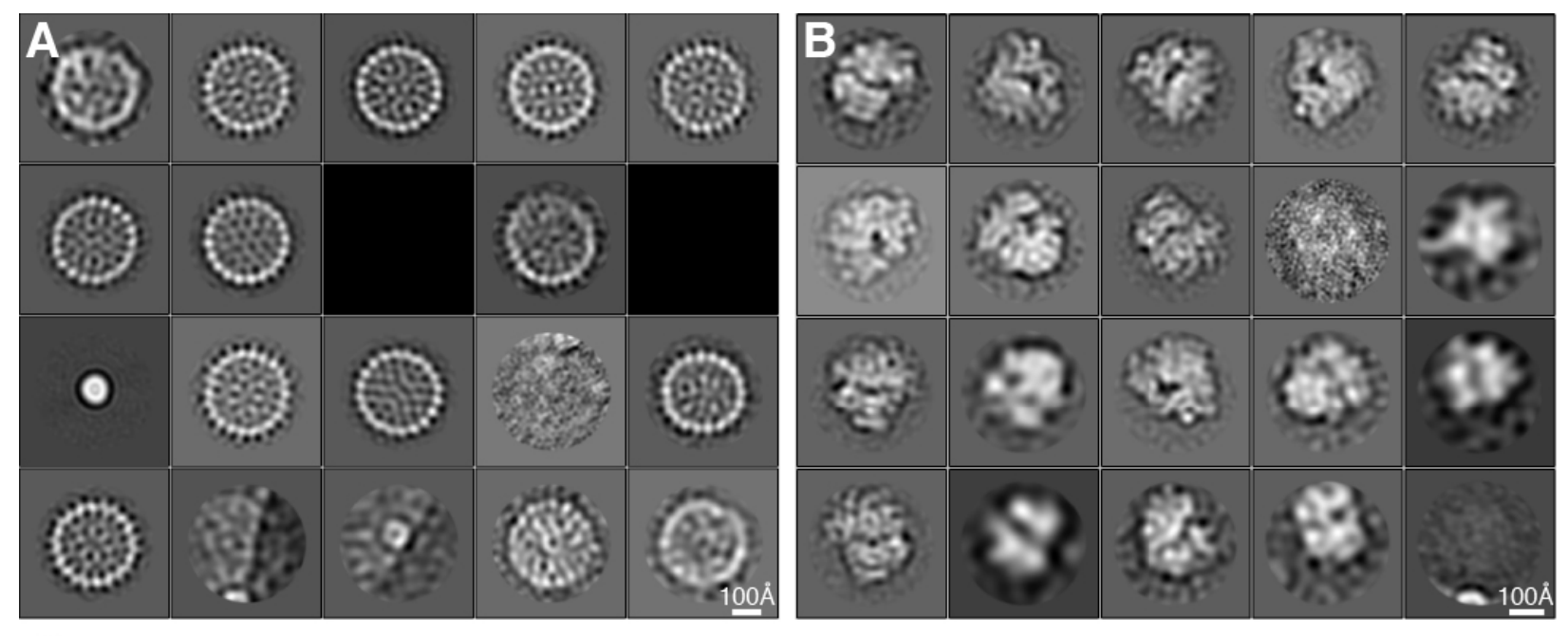

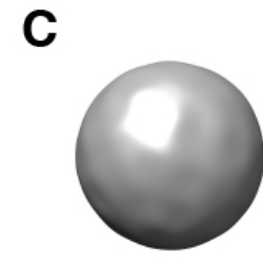

iteration 0

D

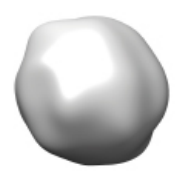

iteration 0

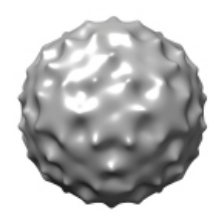

iteration 3

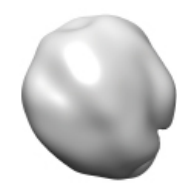

iteration 3

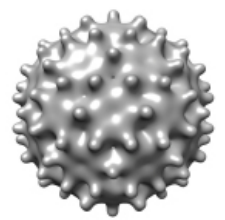

iteration 6

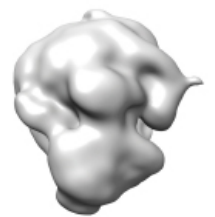

iteration 6

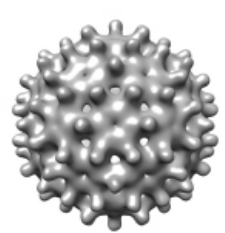

iteration 10

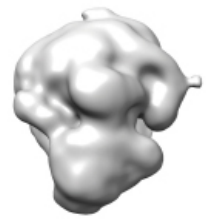

iteration 10

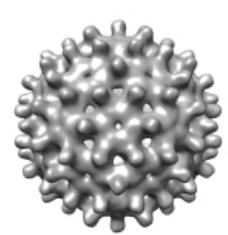

iteration 20

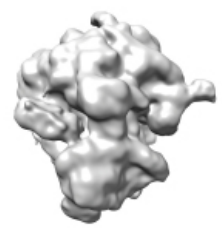

iteration 20

Figure 4. 2D Classification and initial model generation.

(A) 2D classification of projected sub-tomograms of the HBV capsid particles. Particles were selected from a template matching procedure and this classification helps in removing bad particles, for example ones that correspond to $10 \mathrm{~nm}$ gold fiducials. (B) 2D classification of projected sub-tomograms of $S$. cerevisiae $80 \mathrm{~S}$ ribosomes. These data were picked manually in IMOD. (C) Reference-free refinement of the HBV capsid data set. Sub-tomograms were assigned random Euler angles initially (in iteration 0) and then refinement was commenced. (D) Reference-free refinement of the $S$. cerevisiae $80 \mathrm{~S}$ ribosome particles, again starting from random orientations. 


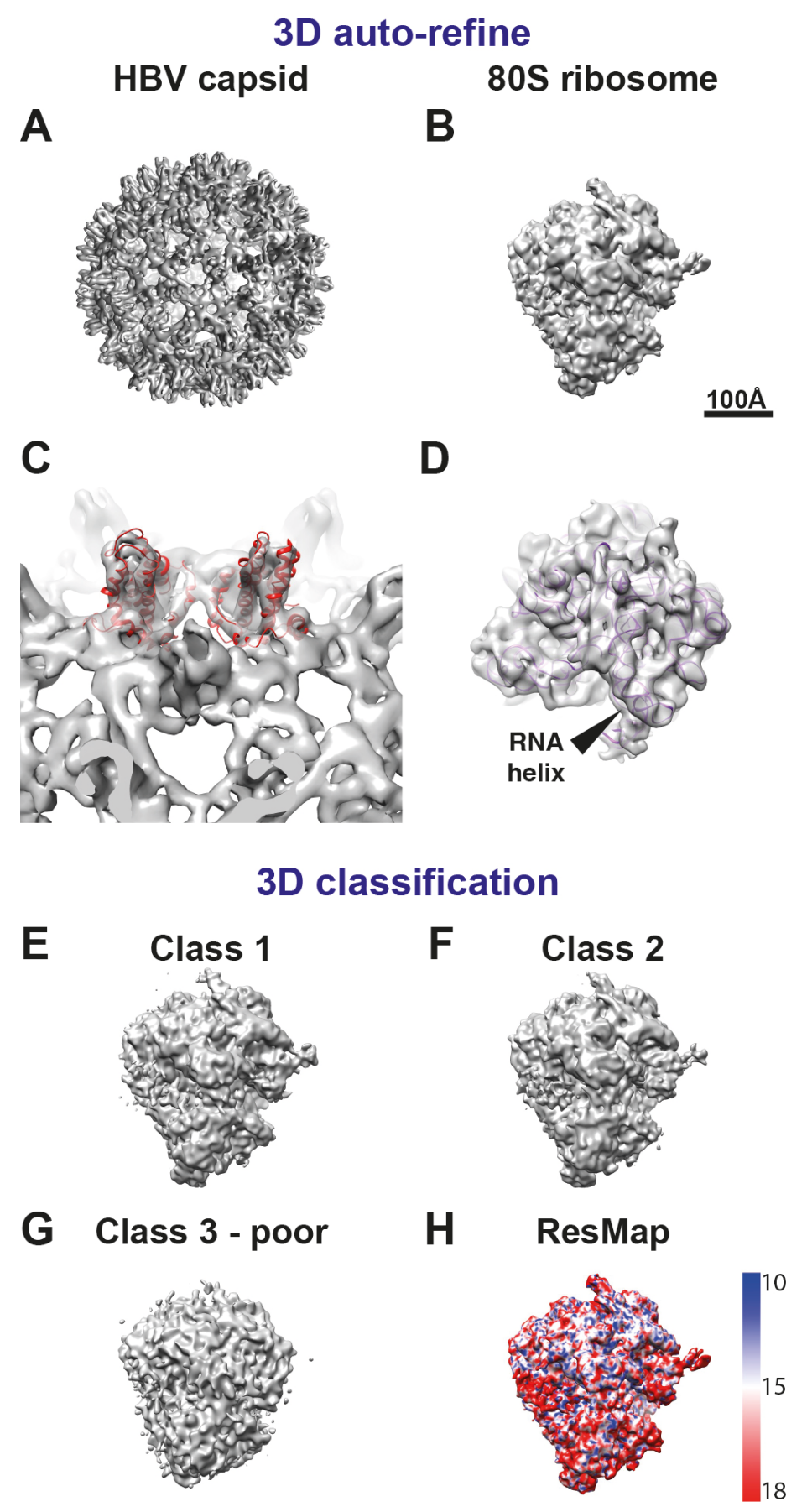

Figure 5. 3D auto-refinement and classification using the regularized-likelihood algorithm in RELION.

(A) Output of 3D auto-refinement procedure from RELION for the HBV capsid dataset. (B) Output of 3D auto-refinement for the 80S ribosome data set (This map has been deposited at the EMDB under the accession number EMD-3228). (C) Secondary structure features ( $\alpha$-helices) are 
resolved in the HBV capsid map. Fitted atomic co-ordinates into the sub-tomogram average highlight the positions of the helices. (D) RNA helices resolved in the $80 \mathrm{~S}$ ribosome map. The atomic co-ordinates have been fitted into this map as rigid bodies for visualization. (E-G) 3D classification of the ribosome data set into three classes reveals a subset of particles $(\sim 15 \%$ of the data set) in $\mathrm{G}$ that show a poor sub-tomogram average. $(\mathrm{H})$ Removing these particles in a second 3D auto-refinement leads to a cleaner map. The result of ResMap is plotted onto the final density showing somewhat lower resolution in the small subunit of the ribosome. 


\section{References}

Agulleiro, J.I., and Fernandez, J.J. (2011). Fast tomographic reconstruction on multicore computers. Bioinformatics 27, 582-583.

Bai, X.C., McMullan, G., and Scheres, S.H. (2015). How cryo-EM is revolutionizing structural biology. Trends Biochem Sci 40, 49-57.

Bartesaghi, A., Lecumberry, F., Sapiro, G., and Subramaniam, S. (2012). Protein secondary structure determination by constrained single-particle cryo-electron tomography. Structure 20, 2003-2013.

Baumeister, W. (2002). Electron tomography: towards visualizing the molecular organization of the cytoplasm. Curr Opin Struct Biol 12, 679-684.

Beck, M., Lucić, V., Förster, F., Baumeister, W., and Medalia, O. (2007). Snapshots of nuclear pore complexes in action captured by cryo-electron tomography. Nature 449, 611-615.

Bharat, T.A., Castillo Menendez, L.R., Hagen, W.J., Lux, V., Igonet, S., Schorb, M., Schur, F.K., Krausslich, H.G., and Briggs, J.A. (2014). Cryo-electron microscopy of tubular arrays of HIV-1 Gag resolves structures essential for immature virus assembly. Proc Natl Acad Sci U S A $111,8233-8238$.

Bharat, T.A., Davey, N.E., Ulbrich, P., Riches, J.D., de Marco, A., Rumlova, M., Sachse, C., Ruml, T., and Briggs, J.A. (2012). Structure of the immature retroviral capsid at $8 \AA$ resolution by cryo-electron microscopy. Nature 487, 385-389.

Bharat, T.A., Russo, C.J., Lowe, J., Passmore, L.A., and Scheres, S.H. (2015). Advances in Single-Particle Electron Cryomicroscopy Structure Determination applied to Sub-tomogram Averaging. Structure 23, 1743-1753.

Briggs, J.A. (2013). Structural biology in situ--the potential of subtomogram averaging. Curr Opin Struct Biol 23, 261-267.

Briggs, J.A., Riches, J.D., Glass, B., Bartonova, V., Zanetti, G., and Kräusslich, H.G. (2009). Structure and assembly of immature HIV. Proc Natl Acad Sci U S A 106, 11090-11095.

Castano-Diez, D., Kudryashev, M., Arheit, M., and Stahlberg, H. (2012). Dynamo: a flexible, user-friendly development tool for subtomogram averaging of cryo-EM data in highperformance computing environments. J Struct Biol 178, 139-151.

Chen, S., McMullan, G., Faruqi, A.R., Murshudov, G.N., Short, J.M., Scheres, S.H., and Henderson, R. (2013). High-resolution noise substitution to measure overfitting and validate resolution in 3D structure determination by single particle electron cryomicroscopy. Ultramicroscopy 135, 24-35.

Cheng, Y. (2015). Single-Particle Cryo-EM at Crystallographic Resolution. Cell 161, 450-457.

Crowther, R.A., Henderson, R., and Smith, J.M. (1996). MRC image processing programs. J Struct Biol 116, 9-16.

Eibauer, M., Hoffmann, C., Plitzko, J.M., Baumeister, W., Nickell, S., and Engelhardt, H. (2012). Unraveling the structure of membrane proteins in situ by transfer function corrected cryo-electron tomography. J Struct Biol 180, 488-496.

Förster, F., Medalia, O., Zauberman, N., Baumeister, W., and Fass, D. (2005). Retrovirus envelope protein complex structure in situ studied by cryo-electron tomography. Proc Natl Acad Sci U S A 102, 4729-4734.

Grünewald, K., Desai, P., Winkler, D.C., Heymann, J.B., Belnap, D.M., Baumeister, W., and Steven, A.C. (2003). Three-dimensional structure of herpes simplex virus from cryo-electron tomography. Science 302, 1396-1398. 
Hall, S.R. (1991). The Star File - a New Format for Electronic Data Transfer and Archiving. J Chem Inf Comp Sci 31, 326-333.

Henderson, R., Sali, A., Baker, M.L., Carragher, B., Devkota, B., Downing, K.H., Egelman, E.H., Feng, Z., Frank, J., Grigorieff, N., et al. (2012). Outcome of the first electron microscopy validation task force meeting. Structure 20, 205-214.

Heymann, J., and Belnap, D. (2007). Bsoft: image processing and molecular modeling for electron microscopy. J Struct Biol 157, 3-18.

Hrabe, T., Chen, Y., Pfeffer, S., Cuellar, L.K., Mangold, A.V., and Forster, F. (2012). PyTom: a python-based toolbox for localization of macromolecules in cryo-electron tomograms and subtomogram analysis. J Struct Biol 178, 177-188.

Huiskonen, J.T., Hepojoki, J., Laurinmaki, P., Vaheri, A., Lankinen, H., Butcher, S.J., and Grunewald, K. (2010). Electron cryotomography of Tula hantavirus suggests a unique assembly paradigm for enveloped viruses. J Virol 84, 4889-4897.

Kremer, J.R., Mastronarde, D.N., and McIntosh, J.R. (1996). Computer visualization of threedimensional image data using IMOD. J Struct Biol 116, 71-76.

Kucukelbir, A., Sigworth, F.J., and Tagare, H.D. (2014). Quantifying the local resolution of cryo-EM density maps. Nature methods 11, 63-65.

Lin, J., Okada, K., Raytchev, M., Smith, M.C., and Nicastro, D. (2014). Structural mechanism of the dynein power stroke. Nat Cell Biol 16, 479-485.

Mindell, J.A., and Grigorieff, N. (2003). Accurate determination of local defocus and specimen tilt in electron microscopy. J Struct Biol 142, 334-347.

Nicastro, D., Schwartz, C., Pierson, J., Gaudette, R., Porter, M.E., and McIntosh, J.R. (2006). The molecular architecture of axonemes revealed by cryoelectron tomography. Science 313, 944-948.

Patwardhan, A., Ashton, A., Brandt, R., Butcher, S., Carzaniga, R., Chiu, W., Collinson, L., Doux, P., Duke, E., Ellisman, M.H., et al. (2014). A 3D cellular context for the macromolecular world. Nat Struct Mol Biol 21, 841-845.

Pettersen, E.F., Goddard, T.D., Huang, C.C., Couch, G.S., Greenblatt, D.M., Meng, E.C., and Ferrin, T.E. (2004). UCSF Chimera--a visualization system for exploratory research and analysis. J Comput Chem 25, 1605-1612.

Pfeffer, S., Burbaum, L., Unverdorben, P., Pech, M., Chen, Y., Zimmermann, R., Beckmann, R., and Forster, F. (2015). Structure of the native Sec61 protein-conducting channel. Nature communications 6,8403 .

Rigort, A., and Plitzko, J.M. (2015). Cryo-focused-ion-beam applications in structural biology. Arch Biochem Biophys 581, 122-130.

Rosenthal, P.B., and Henderson, R. (2003). Optimal determination of particle orientation, absolute hand, and contrast loss in single-particle electron cryomicroscopy. J Mol Biol 333, 721745.

Scheres, S.H. (2012a). A Bayesian view on cryo-EM structure determination. J Mol Biol 415, 406-418.

Scheres, S.H. (2012b). RELION: implementation of a Bayesian approach to cryo-EM structure determination. J Struct Biol 180, 519-530.

Scheres, S.H. (2014). Beam-induced motion correction for sub-megadalton cryo-EM particles. Elife 3, e03665.

Scheres, S.H., and Chen, S. (2012). Prevention of overfitting in cryo-EM structure determination. Nature methods 9, 853-854. 
Schur, F.K., Hagen, W.J., Rumlova, M., Ruml, T., Muller, B., Krausslich, H.G., and Briggs, J.A. (2015). Structure of the immature HIV-1 capsid in intact virus particles at 8.8 A resolution. Nature 517, 505-508.

Tang, G., Peng, L., Baldwin, P.R., Mann, D.S., Jiang, W., Rees, I., and Ludtke, S.J. (2007). EMAN2: an extensible image processing suite for electron microscopy. J Struct Biol 157, 38-46. Yu, L., Snapp, R.R., Ruiz, T., and Radermacher, M. (2013). Projection-based volume alignment. J Struct Biol 182, 93-105. 